\title{
Renal Replacement in End-Stage Renal Disease Patients over 75 Years Old
}

\author{
Isabelle Létourneau Denis Ouimet Marc Dumont Vincent Pichette \\ Martine Leblanc \\ Department of Nephrology, Maisonneuve-Rosemont Hospital and Department of Biostatistics, \\ University of Montreal, Montreal, Quebec, Canada
}

\section{Key Words}

Age $\cdot$ Dialysis $\cdot$ Chronic renal failure $\cdot$ Co-morbidity . Elderly · End-stage renal disease - Hemodialysis .

Peritoneal dialysis $\cdot$ Hospitalization $\cdot$ Survival

\begin{abstract}
Background: Over the last decade, the age of dialysis patients has been increasing steadily in several units in Canada. Our main objective was to assess prevalence, co-morbidity and outcome of ESRD patients over 75 years old at the beginning of dialysis treatment in our center. As a group, they were compared to younger dialysis patients treated simultaneously. Methods: In the last 5 years, all cases beginning dialysis in our institution who were above 75 years of age were reviewed, as well as cases aged between 50 and 60 years who started dialysis during the same period. Between J anuary 1996 and December 2000, among a total of 429 new chronic dialysis patients, 67 ESRD patients over 75 years (15.6\%) and 66 patients between 50 and 60 years (15.4\%) began dialysis treatment. Results - Primary and Secondary: Diabetes was present in $37 \%$ of elderly and in $56 \%$ of the younger patients. Younger patients had been referred earlier to our nephrologists than the older ones (42 vs.
\end{abstract}

$27 \%)$. Elderly were more frequently treated by hemodialysis than peritoneal dialysis (81 vs. $19 \%$ ) when compared to their younger counterparts ( $65 \mathrm{vs.} 35 \%$ ). Longterm catheters for hemodialysis were used more often in elderly patients. No renal transplantation were performed in older patients while 7 younger patients received a renal graft. Survival rates after 1 and 3 years were, respectively, 93 and $74 \%$ for patients between 50 and 60 years, whereas it decreased to 80 and $45 \%$ for those over 75 years $(p=0.002)$. More than $50 \%$ of patients older than 75 years died within 2 years after starting dialysis; their mean survival was 31 months; patients starting dialysis between 50 and 60 years survived on the average 44 months during the study period. According to the multivariate logistic regression model, risk factors for increased mortality in the older group were: number of hospitalization days during the past 3 months (OR 34.8, 95\% Cl 8.3-145.7, p < 0.001) and lower weight (OR 16.6, 95\% Cl 2.0-139.0, p = 0.001). Conclusion: We may conclude that, in our hands, life expectancy of patients who began dialysis above 75 years is significantly shorter than for patients for whom dialysis is initiated between age 50 and 60 years, especially if they have a low weight, lose weight and/or require hospitalization.

Copyright @2003 S. Karger AG, Base
Tel. +1 514252 3489, Fax +1 514255 3026, E-Mail martine.leblanc@sympatico.ca 


\section{Introduction}

Aging of the population is one of the main factors explaining the increase in the number of dialysis patients in recent years in North America. Because of increased life expectancy and better treatment of co-morbidity, we have more and more elderly patients beginning dialysis. In the general population of Canada, life expectancy goes up to 85.1 years in men and to 87.7 years in women who reached 75 years old [1]. What is the outcome of renal replacement therapy in elderly?

In 1990, USRDS reported that $43.5 \%$ of dialysis patients were over 65 years of age whereas $16.3 \%$ were over 75 years [2]. In 2000, the dialysis population over 65 years old was estimated to be near $60 \%$. In 2005, it is estimated that nearly 32,952 patients will receive renal replacement (all age groups included) corresponding to a $85 \%$ increase in prevalence [3].

The objectives of the present study were : (a) to review cases of end-stage renal disease (ESRD) patients beginning dialysis at Maisonneuve-Rosemont Hospital over the last 5 years; (b) to define the prevalence, co-morbidity, risk factors, and outcome of patients over 75 years on renal replacement therapy; (c) to compare co-morbidity and outcome of ESRD patients starting dialysis after 75 years old with those of patients beginning between 50 and 60 years; (d) to evaluate several parameters such as quality of treatment and tolerance to dialysis, nutritional status and, quality of life of our elderly patients on dialysis.

\section{Methods}

Between January 1996 and December 2000, 429 ESRD patients began renal replacement therapy at Maisonneuve-Rosemont Hospital. All cases beginning dialysis who were above 75 years of age were identified and reviewed, as well as cases aged between 50 and 60 years who started dialysis during the same period. A total of 67 ESRD patients had 75 years or more and 66 patients were between 50 and 60 years old.

The following data was recorded: demographics, co-morbidity, medication, cause of ESRD, predicted glomerular filtration rate (GFR) by Cockcroft-Gault formula at the first nephrologist visit, choice of modality and date of first dialysis, change or discontinuation of dialysis modality, type of access, date of creation, and related complications. We defined that patients were referred early if they had met a nephrologist more than 3 months before beginning dialysis [4].

Blood pressure, weight, and several other markers (urea, creatinine, $\mathrm{Kt} / \mathrm{V}, \mathrm{nPCR}$, homocysteine, albumin, pre-albumin, hemoglobin) were also recorded at the first dialysis, at 3 and 6 months and during follow-up until discontinuation of treatment, death or end of the observation period. As per routine, blood/serum is drawn month-
Table 1. Causes of ESRD ${ }^{1}$

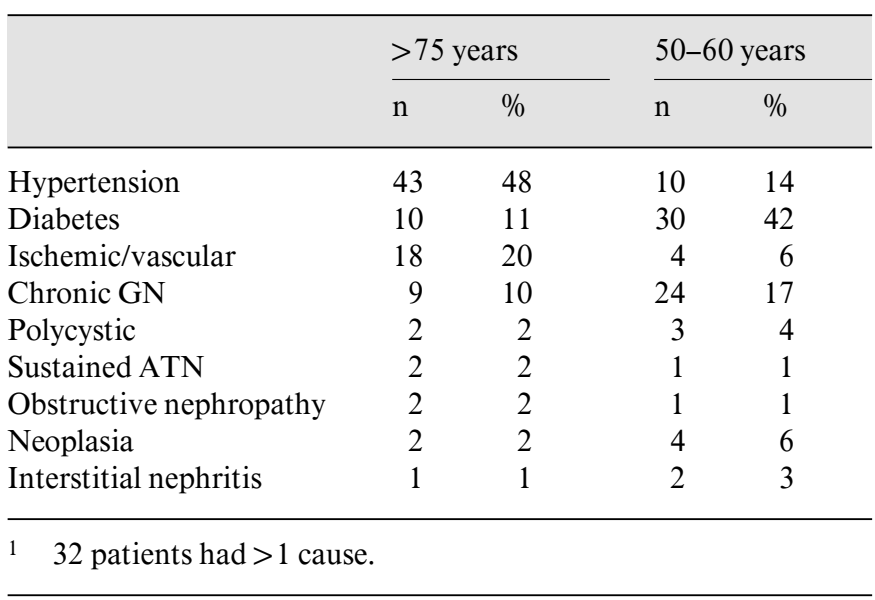

ly in our dialysis patients for laboratory, biochemistry and monitoring of treatment. Biochemistry values recorded in charts at the first and last dialysis were especially considered, including $\mathrm{Kt} / \mathrm{V}$ and $\mathrm{nPCR}$ (in $\mathrm{g} / \mathrm{kg} /$ day), since K-DOQI recommend to follow these variables to ensure adequacy of dialysis [5].

The number of hospitalizations and length of stay, functional status, complaints related to disease, perceptions of health and quality of life, and outcome were all considered. Charts were reviewed extensively by one investigator looking in medical, nursing and social worker notes for patients who complained of any symptoms related to dialysis and for their perception of health, functional status or quality of life. The Karnofsky score or other quality of life scale was not used because of missing information during the review process.

Statistical analysis was performed using $\chi^{2}, \mathrm{t}$ test and stepwise block Cox regression. A multivariate logistic regression model was applied to find risk factors influencing survival in the two groups and between them, with odds ratios (OR) adjusted for confounding variables. We used Kaplan-Meier curves to find a mean survival time on dialysis for both groups. $p$ was found significant if $<0.05$.

\section{Results}

Between January 1996 and December 2000, among a total of 429 new patients who began dialysis treatment in our unit, 67 ESRD were above 75 years of age (15.6\%) and 66 patients were between 50 and 60 years $(15.4 \%)$. Diabetes was present in $37 \%$ of the elderly and in $56 \%$ of the younger group $(p=0.41)$, and was more frequently the cause of ESRD in the patients between 50 and 60 years (table 1). Younger patients had been referred earlier to our nephrologists than the older ones (42 vs. $27 \%$, p < $0.05)$. Elderly were more frequently treated by hemodialysis than by peritoneal dialysis ( 81 vs. $19 \%$ ) when compared to their younger counterparts (65 vs. $35 \%)$ ( $p=$ 


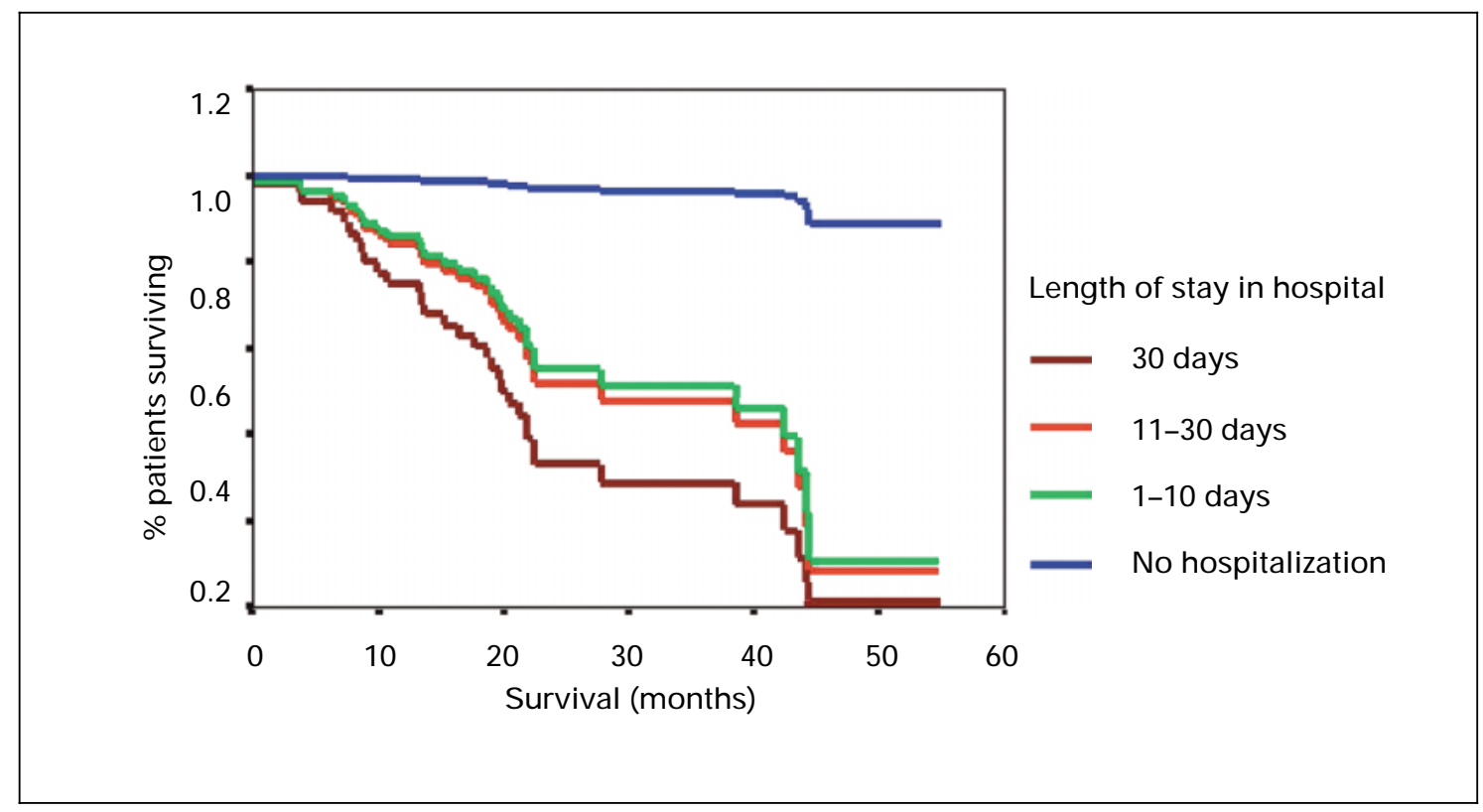

Fig. 1. Survival rate and duration of survival (in months) in relation to number of days of hospitalization.

0.19). Long-term catheters for hemodialysis were used more often in elderly patients ( 31 vs. $21 \%$ in the younger group). Younger patients changed from one modality of treatment to another more often than elderly patients (20 vs. 2 patients). Among the 20 patients between 50 and 60 years old who changed dialysis modality, 7 received a kidney transplant, 5 switched from hemodialysis to peritoneal dialysis and 8 from peritoneal dialysis to hemodialysis. In patients over 75 years old, only 2 patients changed modality, being transferred from hemodialysis to peritoneal dialysis. No renal transplantation was performed in the older group whereas 7 of the younger patients received a renal graft during the observation period.

Survival rates after 1 and 3 years were, respectively, 93 and $74 \%$ for patients between 50 and 60 years, as compared to 80 and $45 \%$ for those over $75(p=0.002)$. More than $50 \%$ of patients older than 75 years died within 2 years after starting dialysis; their median survival was indeed 22 months (95\% CI 5.8-39.2) (mean survival 31 months, 95\% CI 24.9-37.3) (fig. 1). Patients starting dialysis between 50 and 60 years survived on the average 44 months $(95 \%$ CI 39.2-50.4) during the study period ( $\mathrm{p}=$ $0.0024)$. In patients over 75 years, those who died had a higher number of hospitalizations $(4.4 \pm 3.0$ vs. $1.7 \pm$ $1.7, \mathrm{p}<0.001)$ and a much longer stay in hospital $(86.3 \pm$ 62.9 vs. $29.2 \pm 32.4, \mathrm{p}<0.001)$ when compared to survivors. Among the 14 patients between 50 and 60 years old who died, 7 (50\%) had been referred early and 7 (50\%) late, as compared to the 22 non-survivors elderly group of whom $4(25 \%)$ had been referred early and $18(41 \%)$ late $(p=N S)$. Mortality rates in hemodialysis patients versus peritoneal dialysis patients were similar in each group and between them. Patients who maintained some residual diuresis ( $\geq 500 \mathrm{ml}$ per day) seemed to have more chance to survive. In patients between 50 and 60 years old, $31 \%$ of non-survivors maintained their diuresis vs $64 \%$ of survivors ( $p=0.035$ ); in the group over 75 years old only a trend was observed ( $73 \%$ of non-survivors vs. $83 \%$ of survivors, $\mathrm{p}=0.27$ ).

Comorbid conditions for both groups can be found in table 2. As shown in table 3, for both groups, those who died had a poor perception of their health, functional status, and quality of life. Among the 27 non-survivors over 75 years, 15 decided to discontinue dialysis as compared to 8 among the 14 non-survivors between 50 and 60 years. The decision to discontinue was generally based on a very poor quality of life.

According to the univariate analysis, the last values of urea, hemoglobin, albumin, weight and number of hospitalization (total days in hospital, number of days in hospital in the last 3 and 6 months) were all statistically different between survivors and non-survivors (table 4, 5). From these tables, we see that non-survivors had lost weight during the study period in both groups and that 
Table 2. Co-morbidity in our patients

\begin{tabular}{|c|c|c|c|c|c|}
\hline & \multicolumn{2}{|c|}{ Survivors } & \multicolumn{2}{|c|}{ Non-survivors } & \multirow[t]{2}{*}{$\mathrm{p}$} \\
\hline & $\mathrm{n}$ & $\%$ & $\mathrm{n}$ & $\%$ & \\
\hline 75 years and older & 40 & & 27 & & \\
\hline Coronary artery disease & 22 & 55 & 21 & 78 & 0.05 \\
\hline Pulmonary edema ${ }^{1}$ & 6 & 15 & 13 & 48 & 0.003 \\
\hline Hypertension & 37 & 93 & 26 & 96 & NS \\
\hline Diabetes & 15 & 38 & 10 & 37 & NS \\
\hline Peripheral vascular disease & 20 & 50 & 23 & 85 & 0.002 \\
\hline Obstructive pulmonary disease & 10 & 25 & 7 & 26 & NS \\
\hline Neoplasia & 13 & 33 & 8 & 30 & NS \\
\hline Between 50 and 60 years & 52 & & 14 & & \\
\hline Coronary artery disease & 27 & 52 & 7 & 50 & NS \\
\hline Pulmonary edema ${ }^{1}$ & 9 & 17 & 6 & 43 & 0.05 \\
\hline Hypertension & 49 & 94 & 12 & 86 & NS \\
\hline Diabetes & 30 & 58 & 7 & 50 & NS \\
\hline Peripheral vascular disease & 14 & 27 & 5 & 36 & NS \\
\hline Obstructive pulmonary disease & 4 & 8 & 2 & 14 & NS \\
\hline Neoplasia & 5 & 10 & 6 & 43 & 0.006 \\
\hline
\end{tabular}

11 or more episode during the observation period.

Table 3. Perception of health in our patients

\begin{tabular}{|c|c|c|c|c|c|}
\hline & \multicolumn{2}{|c|}{ Survivors } & \multicolumn{2}{|c|}{ Non-survivors } & \multirow[t]{2}{*}{$\mathrm{p}$} \\
\hline & $\mathrm{n}$ & $\%$ & $\mathrm{n}$ & $\%$ & \\
\hline 75 years and older & 40 & & 27 & & \\
\hline Altered functional capacity & 7 & 18 & 17 & 63 & $<0.001$ \\
\hline Complaints about health & 2 & 5 & 3 & 11 & NS \\
\hline Health perception (bad) & 1 & 8 & 8 & 80 & $<0.001$ \\
\hline Quality of health (bad) & 0 & 0 & 8 & 80 & $<0.001$ \\
\hline Between 50 and 60 years & 52 & & 14 & & \\
\hline Altered functional capacity & 5 & 10 & 7 & 50 & 0.001 \\
\hline Complaints about health & 2 & 4 & 4 & 29 & 0.01 \\
\hline Health perception (bad) & 0 & 0 & 2 & 50 & 0.002 \\
\hline Quality of health (bad) & 0 & 0 & 2 & 50 & 0.002 \\
\hline
\end{tabular}

ESRD over 75 years weighed $10 \mathrm{~kg}$ less than patients between 50 and 60 years old at initiation and at the end of dialysis. The body mass index (BMI) was also significantly lower in elderly patients as compared to those between 50 and 60 years old, both at the initiation of dialysis and through the end; in addition, there was a significant reduction in BMI in non-survivors of both groups, whereas it remained stable or even increased in survivors (table 4,5$)$.

$\mathrm{Kt} / \mathrm{V}$ and nPCR were similar in both groups throughout the study period as well as serum phosphorus, pre- albumin, cholesterol, as well as GFR at initiation of dialysis. Medication such as erythropoietin, antihypertensive drugs and phosphorus chelators as well as total number of drugs received by these patients were not found different between survivors and non-survivors. According to the multivariate logistic regression model, risk factors for an increased mortality in the older group were the number of days of hospitalization during the past 3 months (OR $34.8,95 \%$ CI 8.3-145.7, $\mathrm{p}<0.001)$ and a lower weight (OR 16.6, 95\% CI 2.0-139.0, $\mathrm{p}=0.001$ ) (table 6; fig. 2). 
Table 4. Characteristics of ESRD patients over 75 years beginning dialysis: survivors versus non-survivors

\begin{tabular}{lccc}
\hline & Survivors & Non-survivors & $\mathrm{p}$ \\
\hline Number & 40 & 27 & \\
Age at beginning & $78.3 \pm 3.1$ & $78.5 \pm 3.1$ & $\mathrm{NS}$ \\
Sex ratio & $19 \mathrm{M} / 21 \mathrm{~F}$ & $16 \mathrm{M} / 11 \mathrm{~F}$ & $\mathrm{NS}$ \\
First blood urea, mmol/1 & $34 \pm 10$ & $29 \pm 9$ & 0.08 \\
Last blood urea, mmol/1 & $19 \pm 5$ & $23 \pm 7$ & 0.004 \\
First creatinine, $\mu \mathrm{mol} / 1$ & $543 \pm 209$ & $545 \pm 140$ & $\mathrm{NS}$ \\
Last creatinine, $\mu \mathrm{mol} / 1$ & $603 \pm 168$ & $583 \pm 146$ & $\mathrm{NS}$ \\
First Hb, g/l & $103 \pm 18$ & $100 \pm 14$ & $\mathrm{NS}$ \\
Last Hb, g/l & $115 \pm 14$ & $104 \pm 13$ & 0.001 \\
First albumin, g/l & $35.9 \pm 6.3$ & $36.6 \pm 5.1$ & $\mathrm{NS}$ \\
Last albumin, g/l & $39.2 \pm 3.5$ & $31.2 \pm 6.1$ & $<0.001$ \\
tHcy, $\mu \mathrm{mol} / \mathrm{l}$ & $22.7 \pm 5.7$ & $18.7 \pm 4.3$ & 0.03 \\
Kt/V & $1.9 \pm 0.4$ & $1.8 \pm 0.5$ & $\mathrm{NS}$ \\
nPCR, g/kg/day & $0.9 \pm 0.2$ & $1.0 \pm 0.2$ & $\mathrm{NS}$ \\
First weight, kg & $64.9 \pm 11.7$ & $64.3 \pm 13.9$ & $\mathrm{NS}$ \\
Last weight, kg & $66.3 \pm 11.1$ & $60.5 \pm 12.8$ & 0.05 \\
First BMI, median & 23.6 & 22.8 & $\mathrm{NS}$ \\
Last BMI, median & 23.4 & 21.5 & 0.03 \\
Days in hospital ${ }^{1}$ & $7.1 \pm 16.1$ & $29.5 \pm 25.4$ & $<0.001$ \\
& & & \\
\hline
\end{tabular}

First $=$ First value at initiation of dialysis; last $=$ last value at end of the study, discontinuation of treatment or death; $\mathrm{Hb}=$ hemoglobin; $\mathrm{tHcy}=$ homocysteine; $\mathrm{Kt} / \mathrm{V}=$ dose of dialysis; $\mathrm{nPCR}=$ normalized protein catabolic rate; $\mathrm{BMI}=$ body mass index.

1 In last 3 months before end of the study or death.

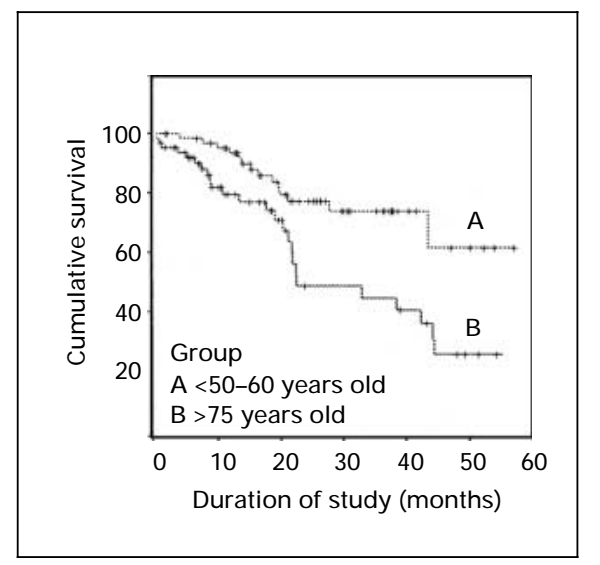

Fig. 2. Cumulative survival in our two groups of patients: A represents the upper curve for patients between 50 and 60 years old (who had a better survival). B represents the lower curve for patients above 75 years old (who had higher mortality).
Table 5. Characteristics of ESRD patients beginning dialysis between 50 and 60 years: survivors versus non-survivors

\begin{tabular}{lccl}
\hline & Survivors & Non-survivors & $\mathrm{p}$ \\
\hline Number & 52 & 14 & \\
Age at beginning & $56.0 \pm 3.1$ & $55.5 \pm 3.0$ & $\mathrm{NS}$ \\
Sex ratio & $31 \mathrm{M} / 21 \mathrm{~F}$ & $6 \mathrm{M} / 8 \mathrm{~F}$ & $\mathrm{NS}$ \\
First blood urea, mmol/1 & $37 \pm 14$ & $33 \pm 11$ & $\mathrm{NS}$ \\
Last blood urea, mmol/1 & $21 \pm 6$ & $22 \pm 8$ & $\mathrm{NS}$ \\
First creatinine, $\mu \mathrm{mol} / 1$ & $778 \pm 428$ & $788 \pm 345$ & $\mathrm{NS}$ \\
Last creatinine, $\mu \mathrm{mol} / 1$ & $684 \pm 204$ & $648 \pm 279$ & $\mathrm{NS}$ \\
First Hb, g/l & $96 \pm 16$ & $93 \pm 18$ & $\mathrm{NS}$ \\
Last Hb, g/l & $115 \pm 17$ & $101 \pm 22$ & 0.009 \\
First albumin, g/l & $35.2 \pm 5.4$ & $34.2 \pm 4.5$ & $\mathrm{NS}$ \\
Last albumin, g/l & $38.5 \pm 4.4$ & $32.0 \pm 7.5$ & 0.007 \\
tHcy, $\mu \mathrm{mol} / \mathrm{l}$ & $23.7 \pm 10.1$ & $18.5 \pm 5.8$ & $\mathrm{NS}$ \\
Kt/V & $1.9 \pm 0.6$ & $1.6 \pm 0.5$ & $\mathrm{NS}$ \\
nPCR, g/kg/day & $1.0 \pm 0.2$ & $0.8 \pm 0.2$ & $\mathrm{NS}$ \\
First weight, kg & $74.8 \pm 17.1$ & $73.9 \pm 16.3$ & $\mathrm{NS}$ \\
Last weight, kg & $78.3 \pm 18.9$ & $66.6 \pm 13.4$ & 0.03 \\
First BMI, median & 26.9 & 26.9 & $\mathrm{NS}$ \\
Last BMI, median & 28.4 & 24.5 & 0.0006 \\
Days in hospital ${ }^{1}$ & $1.7 \pm 5.5$ & $22.1 \pm 19.2$ & 0.002 \\
\hline
\end{tabular}

First $=$ First value at initiation of dialysis; last $=$ last value at end of the study, discontinuation of treatment or death; $\mathrm{Hb}=$ hemoglobin; tHcy = homocysteine; Kt/V = dose of dialysis; $\mathrm{nPCR}=$ normalized protein catabolic rate; $\mathrm{BMI}=$ body mass index

1 In last 3 months before end of the study or death.

Table 6. Factors associated with mortality in our dialysis patients

\begin{tabular}{lcccc}
\hline & Categories & OR & $95 \% \mathrm{CI}$ & $\mathrm{p}$ \\
\hline Age group, years & $50-60$ & 1 & & \\
& 75 and + & 2.66 & $1.30-5.41$ & 0.007 \\
Days in hospital $^{1}$ & 0 & 1 & & \\
& $1-10$ & 18.62 & $4.52-76.73$ & 0.0001 \\
& $11-30$ & 20.54 & $5.33-79.11$ & $<0.0001$ \\
& $>30$ & 34.85 & $8.34-145.68$ & $<0.0001$ \\
Last weight, $\mathrm{kg}^{2}$ & $38-58$ & 16.56 & $1.97-139.0$ & 0.001 \\
& $59-67$ & 8.78 & $1.12-68.65$ & 0.04 \\
& $68-80$ & 9.24 & $1.08-78.83$ & 0.04 \\
& $81-138$ & 1 & &
\end{tabular}

1 In the last 3 months.

2 Last weight recorded before kidney graft, death or at the end of study. 


\section{Discussion}

During the 5 years of study, $15.6 \%$ of patients beginning dialysis were 75 years old and over in our unit. This percentage of elderly is higher than in the study of Munshi [6] in which $9.1 \%$ patients were older than 75 years and $59 \%$ younger than 65 years. A potential explanation is that aging of the population is especially marked in Quebec. However, since the mean age of patients on dialysis in Canada is 56 years old [7], we elected to have a 'control group' between 50 and 60 years to compare their characteristics, evolution and outcome to those of patients above 75 years old.

In our study, $37 \%$ of the elderly were diabetics versus $56 \%$ of younger patients, rates which at first appear similar to those recently published [6]. Since we know that diabetics have an increased mortality rate and a shorter life expectancy, and since we also know that diabetic nephropathy leads to ESRD over a relatively short time frame, it is not surprising to have more diabetics in younger dialysis patients.

Early referral occurred in only $27 \%$ of elderly. Literature recognized that elderly are more frequently referred late since family physicians and nephrologists have oftentimes previously decided that dialysis is not an option for them until symptomatic uremia requires an urgent dialysis or until patients changed their mind at the last minute and want a 'trial of dialysis'. It has been shown that late referral reduces the number of patients choosing peritoneal dialysis and is associated with a greater co-morbidity and higher mortality: lower serum albumin, anemia and lower use of erythropoietin, lower glomerular filtration rate (GFR) at initiation of dialysis, lower rate of functional access and more hospitalization [8].

Elderly were more frequently treated by hemodialysis than by peritoneal dialysis when compared to their younger counterparts, in part because of a higher rate of late referral and probably due to a loss of functional capacity, burden to the family when doing peritoneal dialysis, and better social life in hemodialysis. For vascular access, elderly frequently had a long-term (tunnelized) hemodialysis catheter, probably also because of a late referral and/ or due to vessels of poor quality. Nonetheless, almost $70 \%$ of our elderly had a functional peripheral access (either native or synthetic arteriovenous access).

Patients between 50 and 60 years old changed their modality of treatment more often than elderly patients: it may be explained by a greater number of kidney transplantations in the younger group. In our institution, no renal transplantation was performed in ESRD patients over 75 years after careful evaluation by transplant physicians. Few studies have shown that renal transplantation was an option for ESRD patients over 60 years, showing acceptable graft survival rates attributed to the lower immunocompetence of aging [9]. However, most of the time, elderly with a kidney transplant died soon after receiving a functional graft.

Survival rates were significantly lower in our elderly group since more than $50 \%$ died within 2 years after starting dialysis. Munshi et al. [6] observed survival rates of 53.5 and $2.4 \%$ in ESRD patients over 75 years after 1 and 5 years, respectively as compared to 90.6 and $61.4 \%$ in ESRD patients under 65 years, respectively. In another study [10], survival rates after 1 and 3 years were 78.1 and $48.3 \%$ in ESRD patients over 80 years, thus similar to the rates found in the present study. Röhrich et al. [11] studied 82 ESRD patients over 80 years at initiation of dialysis between 1981 and 1996 and obtained survival rate after 1, 2, 5 and 6 years of dialysis of 70.5, 50.3, 18.5 and $10.6 \%$, respectively. In their hands, elderly beginning dialysis after 1990 had better survival rates than previously (mean of 26 months) [11] possibly consequent to an overall increase in survival with higher delivered dose of dialysis [4]. Also, the Canadian Organ Replacement Registry (CORR) reported that patients over 65 years old have a mortality rate of $43.8 \%$ in non-diabetics and $48 \%$ in diabetics [7].

Mortality has been associated with aging, more than one comorbid factor, malnutrition as defined by a serum albumin $<30 \mathrm{~g} / \mathrm{l}$, inadequate dose of dialysis, longer hospitalization (more than 30 days) [6, 8-12]. By univariate analysis, the same factors seemed to predict death in both groups in our study. Lower serum phosphorus and albumin are recognized risk factors of mortality in dialysis and intensive care unit cohorts. In our study, serum phosphorus was similar in both age groups and also between survivors and non-survivors, whereas serum albumin was significantly reduced in 'final' sampling in non-survivors of both groups. By multivariate analysis, risk factors for increased mortality in our elderly patients were the number of days of hospitalization during the past 3 months and a lower weight. In both subgroups, non-survivors had lost weight during the study period whereas survivors had usually gained a few kilograms. Older patients had a much lower weight and BMI compared to those between 50 and 60 even at dialysis initiation. Small weight was associated to poor survival in both groups. Risk factors of mortality are similar in both groups but being aged over 75 years also confers an increased risk of mortality. Inadequate dose of dialysis does not appear to be a risk factor in our 
population but similar $\mathrm{Kt} / \mathrm{V}$ were delivered in all our patients and it therefore looses its discriminative power. A lower weight may be a sign of malnutrition. Finally, non-survivors of both groups had a bad perception of their health status and quality of life as compared to those surviving.

In conclusion, in our hands, life expectancy of patients who began dialysis above 75 years is significantly shorter than for patients between 50 and 60 years of age. More than $50 \%$ of ESRD over 75 years died within 2 years of the beginning of renal replacement therapy. In both age groups, those who have a lower weight or loose weight and/or require hospitalization are at increased risk of mortality. Unfortunately, we did not find any risk factor of poor outcome before initiation of renal replacement therapy.

\section{References}

1 Statistique Canada, Recueil de statistiques sur la santé et l'état civil 1996, fall 1998, catalogue no 84-214-XPF: Division des statistiques sur la santé - totalisations spéciales.

2 Gomez Campdera FJ, Polo JR, Sanabia J, Tejedor A. First-choice vascular access in patients over 65 years of age starting dialysis. Nephron 1996;73:342-343.

3 Schaubel DE, Morrison HI, Desmeules M, Parsons DA, Fenton SSA: End-stage renal disease in Canada: prevalence projections to 2005. Can Med Assoc J 1999;160:1557-1563.

4 Blake PG, Burkart JM, Churchill DN, Walls J: Early referral needed. Incremental dosing, combined modalities of treatment, and early dialysis initiation were among the topics of discussion during a clinical science symposium at the ASN annual meeting. Nephrology Incite Spring 2000:17-21.
5 National Kidney Foundation-Dialysis Outcomes Quality Initiative: NKF-DOQI clinical practice guidelines for hemodialysis adequacy. Am J Kidney Dis 2001;37(suppl 1):S7-S64.

6 Munshi SK, Vijayakumar N, Taub NA, Bhullar H, Lo TCN, Warwick G: Outcome of renal replacement therapy in the very elderly. Nephrol Dial Transplant 2001;16:128-133.

7 Canadian Institute for Health Information: Canadian Organ Replacement Registry. Center Specific Survival Analysis. Ottawa, Canadian Institute for Health Information, 2000.

8 Arora P, Obrador GT, Ruthazer R, Kausz AT, Meyer KB, Jenuleson CS, Pereira BJG: Prevalence, predictors, and consequences of late nephrology referral at a tertiary care center. J Am Soc Nephrol 1999;10:1281-1286.
9 Stigant CE, Zaltzamn JS: La greffe rénale chez les personnes âgées. Néphrologie 2000;1.

10 Neves PL: Chronic haemodialysis in elderly patients. Nephrol Dial Transplant 1995;10:6971.

11 Röhrich B, Asmus G, von Herrath D, Schaefer $\mathrm{K}$ : Is it worth performing kidney replacement therapy on patients over 80? Nephrol Dial Transplant 1996;11:2412-2413.

12 Merkus MP, Jager KJ, Dekker FW, de Haan RJ, Boeschoten EW, Krediet RT: Predictors of poor outcome in chronic dialysis patients: The Netherlands Cooperative study on the Adequacy of Dialysis. Am J Kidney Dis 2000;35:6979. 